\title{
Prontuário Eletrônico: uma revisão sistemática de implementação sob as diretrizes da Política Nacional de Humanização
}

\author{
Electronic Health Record: a systematic review of the implementation \\ under the National Humanization Policy guidelines
}

Patrícia Pássaro da Silva Toledo (https://orcid.org/0000-0002-4013-7592) ${ }^{1}$ Elizabeth Moreira dos Santos (https://orcid.org/0000-0002-2137-7048) ${ }^{1}$ Gisela Cordeiro Pereira Cardoso (https://orcid.org/0000-0002-4014-0951) ${ }^{1}$ Dolores Maria Franco de Abreu (https://orcid.org/0000-0001-8454-327X) ${ }^{1}$ Alexandre Barbosa de Oliveira (https://orcid.org/0000-0003-4611-1200) ${ }^{2}$
${ }^{1}$ Laboratório de Avaliação (Laser), Departamento de Endemias Samuel Pessoa (DENSP), Escola Nacional de Saúde Pública Sergio Arouca (ENSP), Fundação Oswaldo Cruz (Fiocruz). R. Leopoldo Bulhões 1480, Bonsucesso. 21041-210 Rio de Janeiro RJ Brasil. patriciapassaro@gmail.com ${ }^{2}$ Escola de Enfermagem Anna Nery, Centro de Ciências da Saúde, Universidade Federal do Rio de Janeiro. Rio de Janeiro RJ Brasil.

\begin{abstract}
As part of the evaluability study of the implementation of the Electronic Patient Record (EPR) evaluation, the aim of this Systematic Review (SR) was to identify the evaluation domains to be addressed. This SR, aligned with the Cochrane Handbook for Systematic Reviews of Interventions and the Preferred Reporting Items for Systematic Reviews and Meta-Analyses (PRISMA) encompassed articles published from 2006 to 2019. The search was carried out in the electronic databases SciELO, Oasis IBICT, BVS Regional and Scopus. The search resulted in 1,178 articles, 42 of which met the inclusion criteria. Most studies used qualitative methods for the analyses. The publications took place between 2006 and 2019, with a concentration in 2017 with 9 (21\%) articles published in that year. No studies were published in 2008 and 2009. Only 10 studies included the description, analysis or results related to the domains of implementation. The main domains in which the EPR was problematized were: underutilization; professionals resistance to its use; emphasis on usability; and EPR as an information source. Despite the inclusion of all studies that covered the principles and guidelines of the National Humanization Policy (NHP), they are still incipient.
\end{abstract}

Key words Systematic Review, Electronic Medical Record, Evaluation, Clinical management, Primary Health Care
Resumo Como parte do estudo de avaliabilidade da avaliação da implementação do Prontuário Eletrônico do Paciente (PEP), o objetivo desta Revisão Sistemática (RS) foi identificar os domínios de avaliação a serem abordados. Esta RS, alinhada com o Cochrane Handbook for Systematic Reviews of Interventions e o Preferred Reporting Items for Systematic Reviews and Meta-Analyses (PRISMA) englobou artigos publicados de 2006 até 2019. Realizou-se a busca nas bases de dados eletrônicas SciELO, Oásis IBICT, BVS Regional e Scopus. A busca retornou 1.178 artigos, sendo 42 que atenderam aos critérios de inclusão. A maioria dos estudos utilizaram métodos qualitativos para análises. As publicações ocorreram entre 2006 e 2019, tendo sua concentração em 2017 com $9(21 \%)$ artigos publicados. Não foram identificados estudos publicados em 2008 e 2009. Somente 10 estudos incluíam descrição, análises ou resultados relacionados aos domínios de implementação. Os principais domínios em que o PEP foi problematizado foram: subutilização; resistência dos profissionais ao seu uso; ênfase na usabilidade; e o PEP como repositório de informações. Apesar da inclusão de todos os estudos que contemplaram os princípios e diretrizes da Política Nacional de Humanização (PNH), eles ainda são incipientes.

Palavras-chave Revisão Sistemática, Prontuário Eletrônico, Avaliação, Gestão clínica, Atenção Primária à Saúde 


\section{Introdução}

Este artigo é uma revisão sistemática visando a construção das categorias que serão utilizadas para a avaliação de implementação dos prontuários na atenção primária, sob a perspectiva dos princípios e diretrizes da Política Nacional de Humanização (PNH).

A Sociedade Brasileira de Informática em Saúde considera o sistema de Registro Eletrônico de Saúde (RES) uma tecnologia orientada para as necessidades sanitárias, que une informações sociodemográficas e de assistência de um indivíduo ou grupo social, permitindo o compartilhamento dessas informações entre as instituições de saúde ${ }^{1}$. Tais evidências podem ser utilizadas de diversas maneiras e com diferentes objetivos. Alguns exemplos são os seus usos para fins assistenciais, epidemiológicos e científicos, e ainda para o apoio à tomada de decisão, o planejamento de ações e a implementação de políticas.

Neste estudo adota-se a terminologia "Prontuário Eletrônico do Paciente" (PEP) como exemplo de um RES, tecnologia que, no Sistema Único de Saúde (SUS) permite o registro de características individuais dos usuários, e a consolidação dos dados relativos a agravos e serviços de saúde por atributos de pessoas, grupos e populações, em níveis municipais, regionais, estaduais e nacionais.

A partir da implementação do PEP no SUS, o monitoramento da situação de saúde e da gestão financeira pôde ser qualificado por meio da produção facilitada de relatórios assistenciais, diagnósticos situacionais e estudos epidemiológicos. Apesar de não estar implantado em todos os serviços de saúde do território nacional, existe um esforço do Ministério da Saúde (MS) para a sua institucionalização. Em 2013, o MS lançou o eSUS Atenção Básica, um sistema que contempla informações detalhadas e individualizadas para cada usuário da Atenção Básica à Saúde $(\mathrm{ABS})^{2}$. De acordo com o panorama nacional de implantação de prontuário eletrônico do MS em outubro de 2017, dentre as 42,7 mil Unidades Básicas de Saúde (UBS) em funcionamento distribuídas em 5.564 municípios, apenas 18.284 UBS utilizavam o prontuário eletrônico (3.643 municípios). Nestes municípios o e-SUS foi adotado em 8.764 UBS (2.572 municípios), enquanto 9.520 UBS utilizavam sistemas próprios ou contratados de terceiros ${ }^{3}$. Em 2019, o número de UBS com prontuário eletrônico (e-SUS ou outro PEP) implantado subiu para $23.814^{4}$.

Nesse sentido, torna-se imprescindível compreender os desafios inerentes à implementação desses sistemas. Em levantamento preliminar identificou-se que os estudos sobre a implementação de PEP abordam principalmente questões relacionadas à tecnologia da informação (desenvolvimento de software), custo-efetividade da implementação, utilização epidemiológica e científica dos dados armazenados e desempenho sistêmico desses programas ${ }^{5-7}$. Na busca inicial, questões relacionadas aos princípios e diretrizes da Política Nacional de Humanização, como à transversalidade, à autonomia e protagonismo dos sujeitos, à participação coletiva nos processos de gestão e à corresponsabilização dos diferentes atores implicados no processo de produção de saúde são pouco abordadas na literatura sobre PEP.

Foi realizado um estudo de Revisão Sistemática da Literatura (RS) sobre a implementação do PEP, para identificar os tipos de estudos sobre este instrumento de gestão, no qual buscou-se compreender os principais domínios cognitivos pelos quais os PEP são apreciados, bem como as categorias analíticas utilizadas nesses estudos.

\section{Metodologia}

A RS obedeceu ao protocolo disponibilizado no Cochrane Handbook for Systematic Reviews of Interventions e as diretrizes do Preferred Reporting Items for Systematic Reviews and Meta-Analyses (PRISMA) para a apresentação dos resultados. Foi desenvolvida por meio de sete etapas ${ }^{8,9}$. Inicialmente foi realizada a elaboração do protocolo de pesquisa com a definição de critérios. Em seguida procedeu-se à formulação da pergunta utilizando a estratégia BeHEMoTh ${ }^{10}$. Esta estratégia compreende quatro componentes discriminados para o estudo como: Behaviour of interest a implementação do PEP; Health Context os sistemas de saúde; Exclusions os estudos que não abordam as categorias existentes nas diretrizes e princípios da PNH; e os Models or Theories que incluem as categorias de análises das diretrizes e princípios da PNH organizadas em domínios cognitivos. A pergunta definida foi: quais os domínios cognitivos e as categorias de análise sob a perspectiva da humanização utilizadas nos estudos acerca da implementação do PEP em sistemas de saúde? A terceira etapa incluiu o detalhamento da busca da literatura nas bases de dados apresentando os descritores e as estratégias utilizadas. $\mathrm{O}$ quarto passo abrangeu a seleção e revisão dos estudos com a aplicação dos critérios de inclusão e exclusão. Em seguida foi realizada a análise crítica 
dos artigos. A sexta etapa contemplou a seleção e coleta de dados dos artigos por pares através de instrumentos de validação metodológica. Por último procedeu-se a síntese dos achados agregando-se os estudos de acordo com a semelhança entre eles, referidas às categorias analíticas preestabelecidas no protocolo.

Ressalte-se que o protocolo de pesquisa teve como objetivo fornecer subsídios para a elaboração dos domínios cognitivos e categorias de análise para a avaliação de implementação dos prontuários eletrônicos no SUS.

A busca para realização da RS foi realizada nas bases de dados eletrônicas SciELO, Oásis IBICT, BVS Regional e Scopus em outubro de 2019. Os trabalhos recuperados foram sistematizados e contabilizados em uma planilha eletrônica (software Microsoft Excel), organizados por código do artigo, fonte de pesquisa, string de busca, data da busca, ano de publicação, idioma, título, autor e resumo. Nesta planilha, além dos critérios de exclusão, foram contabilizados e descritos os trabalhos excluídos. As palavras-chave e descritores estão apresentados no Quadro 1. Dada a especificidade de cada base de dados foi necessária a utilização de descritores e palavraschaves diferentes entre as buscas. Cabe ressaltar que a definição das estratégias de busca, das palavras-chave e dos descritores foi realizada após diversas discussões entre os autores, contando com o apoio e orientação técnica da bibliotecária da Biblioteca de Saúde Pública da ENSP/Fiocruz.

O Quadro 2 apresenta os domínios cognitivos e as categorias de análise utilizados. Cabe destacar que os domínios cognitivos são descritos por
Bloom et al. ${ }^{11}$ como processos relacionados ao aprendizado e à compreensão de mundo, e envolvem interpretação e pensamento crítico. Para o autor, domínios cognitivos são construtos que reportam significado ao objeto avaliado. As categorias analíticas são agrupamentos de ideias com características similares ou diferentes que originam uma dada classificação, de forma organizá -las e estruturar as informações a elas referentes ${ }^{12}$. Foram considerados os estudos que abordavam os domínios ou categorias como as funções esperadas para a política de humanização.

Para a exploração dos domínios cognitivos na literatura utilizou-se a análise de conteúdo realizada por meio do software Atlas Ti (versão 7.5.18) onde cada estudo foi explorado pelas categorias contidas no protocolo de pesquisa e detalhadas no Quadro 2.

\section{Resultados}

As diretrizes internacionais do PRISMA foram utilizadas para descrever a condução e os resultados obtidos neste estudo (Figura 1).

$\mathrm{Na}$ etapa de elegibilidade, os 147 artigos foram analisados por dois revisores de maneira independente (um pesquisador externo e a autora principal) que realizaram a leitura na íntegra e a apreciação dos artigos de modo a selecionar aqueles que contemplariam a síntese. Foram sistematizados em uma planilha e organizados de acordo com a ficha de extração de dados que considerou as seguintes informações: ano de publicação; tipo de estudo; objetivos do estudo;

Quadro 1. Registro de buscas bibliográficas.

\begin{tabular}{|c|c|c|}
\hline \multicolumn{3}{|c|}{ Registro de buscas bibliográficas } \\
\hline $\begin{array}{c}\text { Fonte de } \\
\text { Busca }\end{array}$ & Palavras-chave, Descritores e Operadores Booleanos (String) & Resultados \\
\hline $\begin{array}{l}\text { BVS } \\
\text { Regional }\end{array}$ & 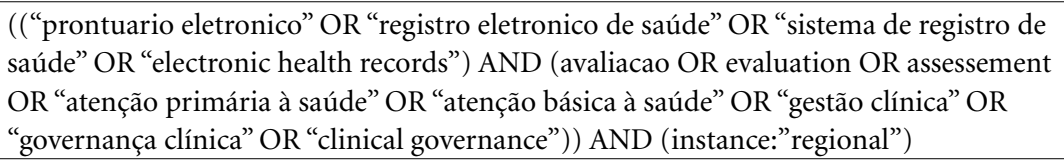 & 494 \\
\hline SciELO & $\begin{array}{l}\text { ("prontuario eletronico" OR “registro eletronico de saude”) AND (avalia* OR "gestao } \\
\text { da clinica”) }\end{array}$ & 33 \\
\hline $\begin{array}{l}\text { OASIS } \\
\text { IBICT }\end{array}$ & $\begin{array}{l}\text { ("prontuario eletronico" OR “registro eletronico de saude") AND (avaliação OR } \\
\text { "gestao da clinica”) }\end{array}$ & 119 \\
\hline Scopus & $\begin{array}{l}\text { (TITLE ("electronic health records") AND TITLE -ABS- } \\
\text { KEY (evaluation OR assessement OR "clinical governance")) }\end{array}$ & 532 \\
\hline \multicolumn{2}{|r|}{ ( } & 1.178 \\
\hline
\end{tabular}

Fonte: Elaborado pelos autores. 
Quadro 2. Domínios cognitivos e categorias analíticas.

\begin{tabular}{|c|c|c|c|}
\hline \multicolumn{2}{|c|}{$\begin{array}{c}\text { Domínios cognitivos (Princípios } \\
\text { e Diretrizes da PNH) }\end{array}$} & \multirow[t]{2}{*}{ Categorias analíticas } & \multirow[t]{2}{*}{ Descrição } \\
\hline Diretrizes & Princípio & & \\
\hline & \multirow[t]{4}{*}{ Transversalidade } & Mudanças de práticas & $\begin{array}{l}\text { Transformação dos modos de relação e de } \\
\text { comunicação entre os sujeitos implicados nos } \\
\text { processos de produção de saúde, produzindo como } \\
\text { efeito a desestabilização das fronteiras dos saberes, } \\
\text { dos territórios de poder e dos modos instituídos na } \\
\text { constituição das relações de trabalho. }\end{array}$ \\
\hline & & $\begin{array}{l}\text { Capacidade } \\
\text { comunicativa intra e } \\
\text { intergrupos }\end{array}$ & $\begin{array}{l}\text { Diálogo ampliado e aberto entre os sujeitos e } \\
\text { coletivos. }\end{array}$ \\
\hline & & $\begin{array}{l}\text { Troca de saberes e } \\
\text { experiências }\end{array}$ & $\begin{array}{l}\text { Troca de saberes e experiências entre os sujeitos e } \\
\text { os coletivos. }\end{array}$ \\
\hline & & $\begin{array}{l}\text { Horizontalização das } \\
\text { relações de trabalho }\end{array}$ & $\begin{array}{l}\text { Organização de uma rede de serviços matriciais } \\
\text { de apoio às equipes de referência. As equipes de } \\
\text { referência em vez de serem um espaço episódico } \\
\text { de integração horizontal passam a ser a estrutura } \\
\text { permanente e nuclear dos serviços de saúde. }\end{array}$ \\
\hline & \multicolumn{2}{|c|}{ Indissociabilidade entre a gestão e a atenção } & $\begin{array}{l}\text { Alteração dos modos de cuidar inseparável da } \\
\text { alteração dos modos de gerir e se apropriar do } \\
\text { trabalho. Inseparabilidade entre clínica e política, } \\
\text { entre produção de saúde e produção de sujeitos. } \\
\text { Integralidade do cuidado e integração dos } \\
\text { processos de trabalho. }\end{array}$ \\
\hline & \multirow[t]{3}{*}{$\begin{array}{l}\text { Protagonismo, } \\
\text { corresponsabilidade } \\
\text { e autonomia dos } \\
\text { sujeitos e coletivos }\end{array}$} & $\begin{array}{l}\text { Democratização das } \\
\text { ações e dos serviços } \\
\text { de saúde }\end{array}$ & $\begin{array}{l}\text { Democratização da gestão dos serviços e } \\
\text { da ampliação da atenção integral à saúde, } \\
\text { promovendo a intra e a intersetorialidade com } \\
\text { responsabilização sanitária pactuada entre gestores } \\
\text { e trabalhadores. }\end{array}$ \\
\hline & & $\begin{array}{l}\text { Corresponsabilização } \\
\text { do cuidado }\end{array}$ & $\begin{array}{l}\text { As mudanças na atenção ganham maior efetividade } \\
\text { quando produzidas pela afirmação da autonomia } \\
\text { dos sujeitos envolvidos, que contratam entre si } \\
\text { responsabilidades compartilhadas nos processos de } \\
\text { cuidar. }\end{array}$ \\
\hline & & $\begin{array}{l}\text { Responsabilização } \\
\text { dos profissionais, } \\
\text { gestores e usuários }\end{array}$ & $\begin{array}{l}\text { As mudanças na gestão ganham maior efetividade } \\
\text { quando produzidas pela afirmação da autonomia } \\
\text { dos sujeitos envolvidos, que contratam entre si } \\
\text { responsabilidades compartilhadas nos processos } \\
\text { de gerir. }\end{array}$ \\
\hline Cogestão & & $\begin{array}{l}\text { Inserção de } \\
\text { novos sujeitos no } \\
\text { planejamento e nos } \\
\text { processos decisórios }\end{array}$ & $\begin{array}{l}\text { Valorização e incentivo para a inclusão dos } \\
\text { trabalhadores e usuários em todo processo de } \\
\text { produção de saúde. }\end{array}$ \\
\hline
\end{tabular}

Fonte: Adaptado dos documentos que compõem a Política Nacional de Humanização ${ }^{13,14}$.

amostra do estudo; metodologia utilizada; domínios cognitivos/categorias de análise; principais resultados encontrados; conclusões do estudo; limitações do estudo; proposições ou recomendações elaboradas.

Os critérios de inclusão abordaram os princípios e diretrizes da PNH, a saber: transversalida- de, indissociabilidade entre a atenção e a gestão; protagonismo, corresponsabilidade e autonomia dos sujeitos e coletivos; e cogestão. Foram incluídos os estudos que: a) discutam a implementação do PEP em relação: às mudanças de práticas e aos modos de interação; ao diálogo ampliado e aberto entre os sujeitos e coletivos; à capacidade co- 


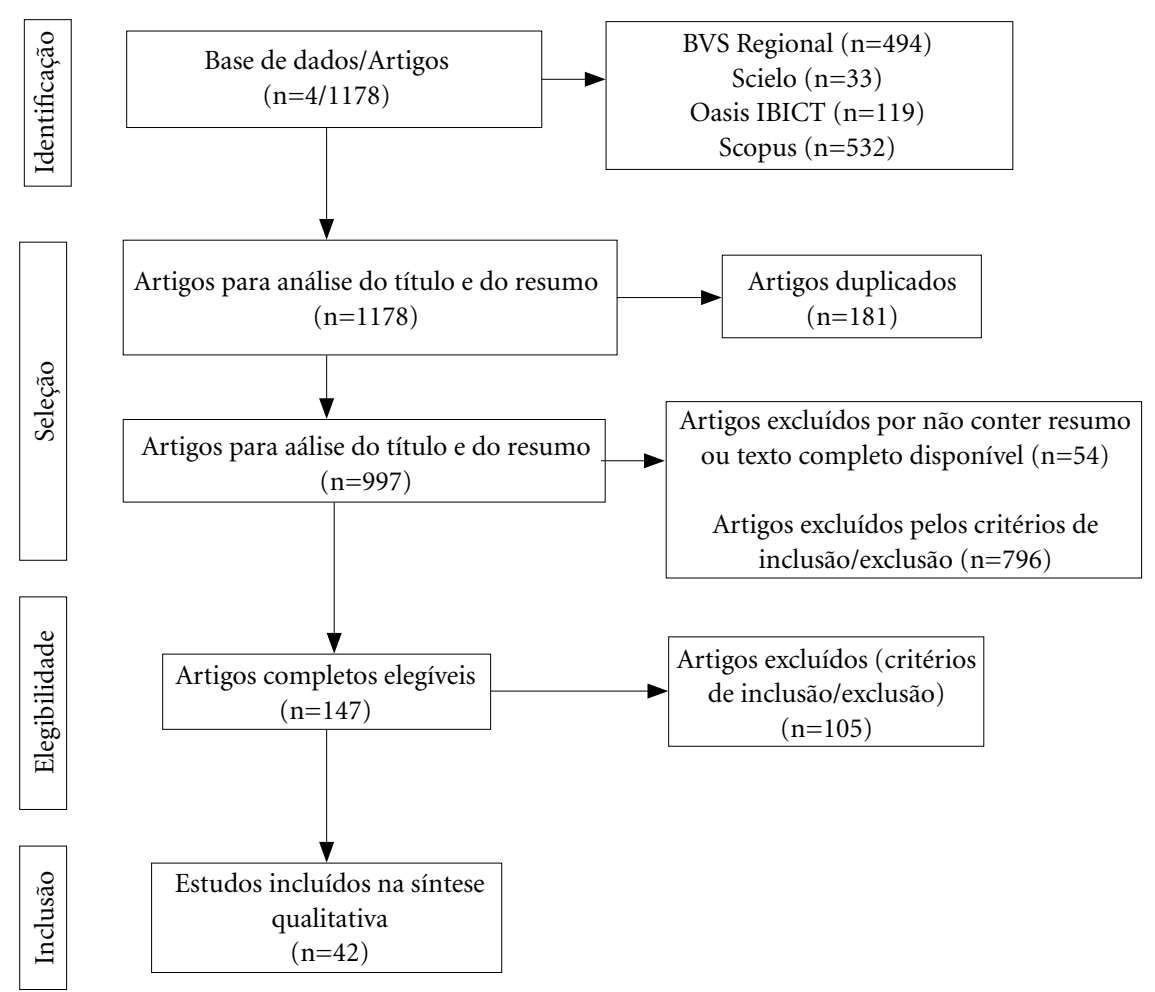

Figura 1. Fluxograma PRISMA.

Fonte: Adaptado de PRISMA Statement ${ }^{15,16}$ pelos autores.

municativa intra e intergrupos; à troca de saberes e experiências; e à horizontalização das relações de trabalho; b) abordem o PEP como um instrumento que favorece ou prejudica a indissociabilidade entre a gestão e a atenção; c) contemplem a discussão do PEP como instrumento de promoção do empoderamento e da autonomia dos sujeitos; da constituição de redes de corresponsabilização pelo cuidado; e da responsabilização dos trabalhadores, gestores e usuários como sujeitos ativos nos processos de gerir e de cuidar; d) discutam o PEP como indutor de espaços coletivos de discussão com a inserção de novos sujeitos no planejamento e nos processos decisórios; como promotor da democratização das ações e dos serviços de saúde ao mesmo tempo que cria corresponsabilidade na gestão do sistema de saúde.

Os critérios de exclusão foram: a) abordarem análises sobre engenharia de software; b) contemplarem análises relativas apenas à módulos complementares ao PEP, tais como portal do paciente, módulo de divulgação de resultado de exames, módulo de apoio à decisão clínica, módulo de busca por fenótipo para pesquisas científicas; c) abordarem o PEP apenas como fonte de dados para o estudo e, portanto, não contemplarem uma análise ampliada sobre o prontuário eletrônico. Foram excluídos nesta etapa os estudos que abordavam questões relacionadas à engenharia de software e mineração de dados.

Previa-se, em caso de discordância, a aplicação de a técnica de consenso, o que não foi necessário pela inexistência de divergências entre os avaliadores.

Para a síntese foram incluídos 42 artigos, sendo 11 em português e 31 em inglês. As publicações ocorreram entre 2006 e 2019, tendo sua concentração em 2017 com nove artigos publicados. A busca não encontrou nenhum artigo publicado nos anos de 2008 e 2009.

Dos estudos incluídos nesta revisão 10 contemplavam análises ou resultados relacionados aos domínios ou categorias propostas, embora alguns artigos apresentassem mais de uma cate- 
goria analítica. No total houve 18 referências às categorias. O Quadro 3 apresenta a distribuição das categorias nos estudos analisados.

Nesta análise 32 estudos não abordaram as categorias analíticas propostas nesta RS.

\section{Discussão}

A categoria mudança de práticas foi abordada em dois estudos. O'Malley et al..$^{18}$ verificaram em sua pesquisa que a adoção do PEP ocasionou mu- dança nas práticas e melhorias assistenciais. Relatam um aumento da "eficiência" percebida pelos profissionais e na satisfação dos médicos em relação ao seu processo de trabalho. Os informantes atribuíram a melhoria na "eficiência" ao prontuário eletrônico favorecer a concentração nas necessidades dos pacientes durante $\mathrm{o}$ atendimento $\mathrm{e}$ direcionar as ações do médico durante a consulta. Schenk et al. ${ }^{20}$ também observaram mudança nas práticas com a adoção do PEP em seus achados. Notou-se mudança significativa no tempo que os enfermeiros dedicavam ao atendimento

Quadro 3. Distribuição das categorias analíticas por artigos incluídos.

\begin{tabular}{|c|c|c|c|}
\hline Categorias Analíticas para Revisão Sistemática & \multicolumn{2}{|c|}{$\begin{array}{l}\text { Artigos que abordaram as categorias analíticas } \\
\text { (como categorias de análise ou em seus achados) }\end{array}$} & Total \\
\hline Mudanças de práticas & \multicolumn{2}{|c|}{$\begin{array}{l}\text { O'Malley et al. } 2015^{18} \\
\text { Schenk et al. } 2018^{20}\end{array}$} & 2 \\
\hline Capacidade comunicativa intra e intergrupos & \multicolumn{2}{|l|}{$\begin{array}{l}\text { Gomes et al. } 2019^{21} \\
\text { Alanazi et al. } 2019^{22} \\
\text { Rathert et al. } 2017^{17} \\
\text { O'Malley et al. } 2015^{18} \\
\text { Sockolow et al. } 2011^{23}\end{array}$} & 5 \\
\hline Troca de saberes e experiências & \multicolumn{2}{|l|}{ Rathert et al. $2017^{17}$} & 1 \\
\hline Horizontalização das relações de trabalho & \multicolumn{2}{|l|}{ O’Malley et al. $2015^{18}$} & 1 \\
\hline Indissociabilidade entre a gestão e a atenção & \multicolumn{2}{|l|}{$\begin{array}{l}\text { Martins et al. } 2017^{19} \\
\text { Godoy et al. } 2012^{25} \\
\text { Holmes } 2016^{26} \\
\text { Schenk et al. } 2018^{20}\end{array}$} & 4 \\
\hline Democratização das ações e dos serviços de saúde & \multicolumn{2}{|l|}{-} & 0 \\
\hline Corresponsabilização do cuidado & \multicolumn{2}{|l|}{$\begin{array}{l}\text { Martins et al. } 2017^{19} \\
\text { Rathert et al. } 2017^{17} \\
\text { O'Malley et al. } 2015^{18} \\
\text { Morrison et al. } 2013^{27}\end{array}$} & 4 \\
\hline $\begin{array}{l}\text { Responsabilização dos profissionais, gestores e } \\
\text { usuários }\end{array}$ & \multicolumn{2}{|l|}{-} & 0 \\
\hline $\begin{array}{l}\text { Inserção de novos sujeitos no planejamento e nos } \\
\text { processos decisórios }\end{array}$ & \multicolumn{2}{|l|}{ Rathert et al. $2017^{17}$} & 1 \\
\hline $\begin{array}{l}\text { Não abordaram as categorias analíticas propostas } \\
\text { para este estudo }\end{array}$ & $\begin{array}{l}\text { Mcbride et al. } 2017 \\
\text { Yanamadala et al. } 2016 \\
\text { Mundim et al. } 2016 \\
\text { Gonçalves et al. } 2013 \\
\text { Welch et al. } 2007 \\
\text { Paek et al. } 2006 \\
\text { Fumis et al. } 2014 \\
\text { Lin et al. } 2019 \\
\text { Kuo et al. } 2018 \\
\text { Hagglund et al. } 2017 \text { a } \\
\text { Hagglund et al. } 2017 \mathrm{~b} \\
\text { Tharmalingam et al. } 2016 \\
\text { Pandit et al. } 2013 \\
\text { Hiligoss et al. } 2013 \\
\text { Greenhalgh et al. } 2010 \\
\text { Graetz et al. } 2015\end{array}$ & $\begin{array}{l}\text { Kami et al. } 2015 \\
\text { Joynt et al. } 2015 \\
\text { Bhuyan et al. } 2014 \\
\text { Lima et al. } 2018 \\
\text { Colleti Junior et al. } 2018 \\
\text { Souza et al. } 2017 \\
\text { Farias et al. } 2014 \\
\text { Graham et al. } 2018 \\
\text { Ericson et al. } 2017 \\
\text { Duarte et al. } 2017 \\
\text { Plantier et al. } 2017 \\
\text { Mysen et al. } 2016 \\
\text { Ser et al. } 2014 \\
\text { Takian et al. } 2014 \\
\text { Silverman et al. } 2014 \\
\text { Stewart et al. } 2010\end{array}$ & 32 \\
\hline
\end{tabular}


individual de pacientes em relação às outras atividades. Adicionalmente, o estudo, identificou que, embora os enfermeiros tenham utilizado mais tempo em intervenções de enfermagem em geral, como por exemplo na administração de medicamentos, tiveram menos de interação com pacientes e suas famílias, especialmente em atividades de educativas e de orientação. Os autores observaram ainda que os enfermeiros demonstraram uma ligeira redução na eficácia do cuidado percebida após a adoção do $\mathrm{PEP}^{20}$. Mudanças que devem ser melhor exploradas em estudos posteriores para maior compreensão das suas influências nas ações de educação em saúde.

Cinco estudos abordaram a categoria capacidade comunicativa intra e intergrupos. Uma pesquisa realizada na Atenção Básica de um município de Minas Gerais descreveu o PEP como uma ferramenta de integração de equipe e cuidado ${ }^{21}$. Outro estudo o apontou como um instrumento útil para aprimorar a comunicação entre os profissionais de saúde ${ }^{22}$. O terceiro estudo, uma revisão sistemática, indicou que o uso do PEP facilita a comunicação e a troca de informações entre a equipe, mas apontou a necessidade de estudos mais robustos que expliquem como essa transformação influencia as funções da comunicação ${ }^{17}$. Em outro estudo, os entrevistados descreveram que a comunicação aprimorada pelo PEP em uma equipe ocorre por meio de acesso e compartilhamento de informações do paciente mobilizando diversos recursos, como mensagens instantâneas no próprio sistema, anotações dentro do prontuário, lembretes e atribuições de tarefas ${ }^{18}$. O último estudo que abordou o tema identificou nas entrevistas que o principal meio de comunicação da equipe era a comunicação presencial e não o $\mathrm{PEP}^{23}$. Este achado corrobora a discussão apresentada por Alanazi et al. ${ }^{22}$ que ressalta $o$ senso comum sobre a melhoria da comunicação atribuída ao PEP baseado na preconcepção de que o sistema interligando diferentes serviços e departamentos de uma unidade de saúde poderia aprimorar a comunicação organizacional.

A categoria troca de saberes e experiências foi abordada por Rathert et al. ${ }^{17}$ em sua revisão sistemática. Seus achados apontaram a relevância do PEP para a troca de informações biomédicas e psicossociais, sendo essencial tanto para o diagnóstico adequado quanto para o desenvolvimento de planos de tratamento. Cabe destacar que estas informações também podem ser utilizadas para orientar as práticas clínicas. Os estudos que compuseram a revisão relataram a prática médica baseada em evidências, a partir de informações coletadas em prontuários eletrônicos.
A categoria horizontalização das relações de trabalho foi brevemente discutida nos achados de O'Malley et al. ${ }^{18}$. Os participantes do estudo relataram a redefinição das funções da equipe de saúde com a implementação do PEP, a partir da delegação de tarefas que, combinadas com fluxos de trabalho clínicos revisados permitiram que profissionais não médicos tivessem suas funções aprimoradas. Não há registro sobre o efeito nos profissionais médicos da equipe de atendimento.

As categorias mudanças de práticas; capacidade comunicativa intra e intergrupos; troca de saberes e experiências; e horizontalização das relações de trabalho compõem o princípio da transversalidade da $\mathrm{PNH}$, que prevê a ampliação das formas de conexão e a melhoria das relações de trabalho, promovendo as práticas de saúde em rede $^{24}$.

A categoria indissociabilidade entre a gestão e a atenção foi observada em quatro estudos. Martins et al. ${ }^{19}$ ressaltam em seus achados a utilização do prontuário eletrônico como ferramenta de gerenciamento das unidades de saúde, e permite uma visão administrativa e sanitária, abrangente, da situação de saúde da unidade. Em outra pesquisa $^{20}$ notou-se que os enfermeiros passaram significativamente mais tempo realizando ações relacionadas ao gerenciamento como coordenação, planejamento de ações e à tomada de decisões. Nas entrevistas, os participantes enfatizaram o papel significativo do PEP como ferramenta de gestão, para o planejamento e tomada de decisão, visto que engloba a maioria das ações de saúde realizadas no âmbito dos equipamentos de saúde. A partir dos registros de atendimentos e atividades, o sistema compila todas as informações necessárias para a composição do prontuário clínico dos usuários, bem como todas as informações necessárias aos gestores da Saúde Pública ${ }^{25}$. No entanto, em uma pesquisa realizada nas unidades da Estratégia Saúde da Família (ESF) em João Pessoa-PB verificou-se que a utilização das fichas do e-SUS era precária, isto é, os profissionais pouco utilizam os dados provenientes deste sistema para tomar decisões em suas práticas. Os participantes do estudo também afirmaram que a gestão emprega pouco as informações coletadas por meio do e-SUS para o planejamento e a execução de ações em saúde. Os informantes atribuem a baixa utilização destes dados às dificuldades inerentes à usabilidade do sistema, à falta de treinamento para operacionalização do prontuário eletrônico e à inadequação do $\mathrm{PEP}$ à realidade local $^{26}$.

A categoria corresponsabilização do cuidado foi abordada em quatro estudos. Martins et al. ${ }^{19}$ 
observaram em um município do sul do Brasil que os profissionais lotados nas Unidades Básicas de Saúde (UBS) utilizaram o PEP durante o processo de trabalho. Os profissionais relataram que usaram o sistema de prontuário eletrônico em todas as suas atividades como ferramenta de consulta para as atividades diárias de sua responsabilidade e para o planejamento de ações para as atividades das equipes de saúde. Rathert et al. ${ }^{17}$ verificaram que o PEP auxilia na elaboração do diagnóstico, na elaboração de plano terapêutico e no desenvolvimento de planos de tratamento para pacientes complexos, facilitando a discussão para a tomada de decisão com os próprios pacientes. O'Malley et al. ${ }^{18}$ apresentam como exemplo o acompanhamento de alguns pacientes com necessidades terapêuticas complexas. Os farmacêuticos coletam as informações inseridas no PEP por outros profissionais subsidiando as atividades de educação em saúde que estes profissionais realizam quando da dispensação dos medicamentos. Os entrevistados explicaram que o PEP oferece suporte a orientações permanentes e protocolos integrados. Consideram que isto contribui para o aumento de autonomia dos membros da equipe, especialmente para o acompanhamento pela enfermagem de ações como vacinações ou exames de saúde que precedem o atendimento médico ${ }^{18}$. Em outro estudo ${ }^{27}$ observaram que a tecnologia disponível nos sistemas de prontuário eletrônico estava suscitando o desejo dos pacientes por informações, aumentando a proatividade em seus cuidados de saúde e melhorando adesão ao tratamento. Isso é especialmente válido em casos de doenças crônicas, como o diabetes. Os participantes afirmaram que o gerenciamento de medicamentos era uma área que poderia se beneficiar muito com o fornecimento de informações estruturadas para e dos usuários, permitindo uma maior compreensão dos cuidados necessários e maior adesão aos medicamentos.

A categoria inserção de novos sujeitos no planejamento e nos processos decisórios só foi identificada em um dos estudos analisados. Trata-se de uma revisão sistemática realizada por Rathert et al. ${ }^{17}$ na qual um dos estudos postulou que o PEP auxilia a elaboração do diagnóstico, a tomada de decisão e o desenvolvimento de planos de tratamento para pacientes complexos, facilitando a discussão para a tomada de decisão com os próprios pacientes. Dessa forma, os autores afirmam que o uso de PEP oferece oportunidades para o paciente e o médico compartilharem o poder. Este estudo também demonstrou a relevância do PEP para a troca de informações biomédicas e psicossociais entre os profissionais e pacientes, sendo essencial tanto para o diagnóstico adequado quanto para o desenvolvimento de planos de tratamento compartilhados.

O quadro de distribuição de categorias (Quadro 3 ) descreve os estudos que, apesar de incluir o uso de prontuários eletrônicos, não abordaram as categorias da PNH. O detalhamento dos mesmos é importante para caracterizar o tipo de categorias mais abrangidas.

Além dos 42 estudos identificados, foram analisados dois estudos não incluídos nesta revisão por não atenderem aos critérios de busca definidos. Estes reforçam a importância do PEP na Atenção Primária à Saúde (APS) em relação aos seus atributos essenciais ${ }^{28,29}$. A longitudinalidade da atenção, a integralidade da assistência e a coordenação do cuidado são atributos que podem ser favorecidos com a implementação de prontuários que permitam a comunicação intra e intergrupos, favorecendo a cogestão e a mudança de práticas.

\section{Considerações finais}

Estudos que contemplam a análise da implementação do PEP são publicados anualmente. Abordam, em sua maioria, critérios de usabilidade, satisfação do usuário, relação custo-benefício e mudanças nos resultados de saúde em relação à implementação desta tecnologia. Estes estudos contribuem para a identificação dos benefícios e dos desafios de sua implementação.

Os artigos apontaram certa resistência na utilização do PEP por parte dos profissionais atribuída à falta de capacitação para sua utilização. Observou-se um investimento em treinamentos com foco na usabilidade e ênfase ao manuseio do sistema. Em sistemas de saúde onde o uso do PEP é obrigatório e cujos profissionais já participaram de treinamentos de usabilidade, notou-se a subutilização dos prontuários, sendo mobilizados apenas como um repositório de informações dos pacientes para gestão clínica dos casos e acompanhamento epidemiológico. Esta resistência deve ser aprofundada em estudos que analisem as mudanças de práticas implicadas nos processos de produção de saúde e sua relação com a implementação do prontuário eletrônico. A compreensão deste processo pode potencializar a adoção do PEP pelos profissionais de saúde no seu cotidiano.

Outro ponto observado foi o foco na comunicação interprofissional. A capacidade comunicativa intra e intergrupos ainda se limita à troca de informações entre os profissionais de saúde. 
Em relação à troca de saberes os estudos também pontuaram a necessidade de incentivar a adoção do PEP para o compartilhamento de experiências entre a equipe de saúde local, entre ela e a comunidade científica, e com os usuários dos serviços de saúde. A adoção de aplicativos de acesso para os pacientes aos seus dados clínicos pode ser um bom caminho para estimular esta troca de saberes.

A implementação do PEP, de acordo com os estudos analisados, favoreceu a organização de uma rede de serviços com integração horizontal entre a equipe de profissionais, promovendo uma gestão mais democrática e participativa nos serviços de saúde. Neste sentido, o PEP foi apontado como promotor de mudanças nos modos de cuidar e de gerir, sem a dissociação entre a integralidade do cuidado e integração dos processos de trabalho, garantindo a indissociabilidade entre a gestão e a atenção, que é um dos princípios da $\mathrm{PNH}$.

Os estudos também apontaram a influência do PEP na corresponsabilização do cuidado e na produção da autonomia dos profissionais de saúde. No entanto, em relação aos pacientes, esta relação ainda é frágil. Este fato é sintomático, pois pode indicar a ausência de mecanismos nos prontuários eletrônicos que promovam a autonomia dos sujeitos, especialmente no que se refere ao paciente. A inserção de novos sujeitos no planejamento e nos processos decisórios reflete o princípio da cogestão da PNH. Nesta perspectiva, a compreensão do papel do PEP para a adoção de práticas de cogestão ainda não é clara e a literatura ainda é escassa neste sentido.

Com efeito, a discussão sobre o PEP como uma ferramenta que pode favorecer a comuni- cação ativa e ampliada, a transversalidade, a integralidade e o protagonismo dos sujeitos nos processos de produção do cuidado, ainda é incipiente. Poucos são os estudos que analisam a relação da implementação do PEP com o processo de humanização do cuidado, do trabalho e da gestão em saúde. É importante destacar que os princípios e diretrizes da $\mathrm{PNH}$ estão intrinsecamente relacionados aos atributos essenciais da Atenção Primária à Saúde (APS). Estudos brasileiros já demonstraram a relevância do PEP para a APS ao favorecer a coordenação do cuidado, a integralidade da assistência e a longitudinalidade da atenção. Esta discussão é fundamental para que propostas de educação continuada, abordando a relação do PEP com os princípios e diretrizes da PNH e como atributos da APS, sejam implementadas, promovendo a qualificação dos profissionais e permitindo um cuidado mais humanizado.

Dentre as limitações deste estudo aponta-se que geralmente o conceito de humanização em saúde não está pautado em políticas públicas, se considerado o cenário global. Assim, os princípios e diretrizes aqui contempladas nem sempre aparecem relacionadas entre si nas diferentes pesquisas. Outros estudos devem ser elaborados, para permitir compreensão ampliada dos desafios da implementação do PEP, na perspectiva da humanização em saúde.

Esta RS buscou analisar a implementação do PEP sob a perspectiva dos princípios e diretrizes da PNH. Cada categoria forneceu subsídios para a construção de dimensões para a avaliação da implementação do PEP. Esta avaliação fornecerá informações relevantes para a tomada de decisão da gestão na implementação de prontuários eletrônicos SUS.

\section{Colaboradores}

PPS Toledo trabalhou na concepção, metodologia, análise, discussão e redação final. EM Santos trabalhou na concepção, metodologia, discussão e redação final. GCP Cardoso trabalhou na leitura e revisão crítica. $\mathrm{AB}$ Oliveira trabalhou na leitura, revisão crítica e metodologia. DMF Abreu participou como revisora independente dos artigos.

\section{Referências}

1. Sociedade Brasileira de Informática em Saúde (SBIS). Conselho Federal de Medicina (CFM). Cartilha sobre Prontuário Eletrônico: a certificação de sistemas de registro eletrônico de saúde [Internet]. 2012 [acessado 2020 fev 14]. Disponível em: http://portal.cfm.org.br/ crmdigital/Cartilha_SBIS_CFM_Prontuario_Eletronico_fev_2012.pdf. 
2. Brasil. Ministério da Saúde (MS). E-SUS Atenção Básica: Manual Do Sistema Com Coleta de Dados Simplificada: CDS [Recurso Eletrônico]. 2014 [acessado 2020 fev 14]. Disponível em: http://189.28.128.100/ dab/docs/portaldab/documentos/manual_CDS_ ESUS_1_3_0.pdf.

3. Brasil. Ministério da Saúde (MS). Relatório de Gestão 2017 [Internet]. 2018 [acessado 2020 fev 14]. Disponível em: https://www.gov.br/saude/pt-br/centraisde-conteudo/relatorio-de-gestao-2017-completo-para-ms-pdf/view.

4. Brasil. Ministério da Saúde (MS). Relatório de Gestão 2019 [Internet]. 2020 [acessado 2020 fev 14]. Disponível em: https://bvsms.saude.gov.br/bvs/publicacoes/ relatorio_gestao_ministerio_saude_2019.pdf.

5. Canêo PK, Rondina JM. Prontuário Eletrônico do Paciente: conhecendo as experiências de sua implantação. J Health Inform 2014; 6(2):67-71.

6. Jenal S, Évora YDM. Revisão de literatura: implantação de prontuário eletrônico do paciente. $J$ Health Inform 2012; 4(4):176-181.

7. Oliveira JF. Gestão de Tecnologias da Informação e da Comunicação na Saúde: uma análise sobre o uso do prontuário eletrônico. Interface (Botucatu) 2013; 9(1):6-25.

8. Ercole FF, Melo LS, Alcoforado CLGC. Integrative review versus systematic review. Reme Rev Min Enferm 2014; 18(1):9-11.

9. Santos-Filho SB. Indicadores de valorização do trabalho e trabalhadores da saúde: construindo o conceito de valorização a partir de uma perspectiva analítica. In: Trabalhador Da Saúde: Muito Prazer! Protagonismo Dos Trabalhadores Na Gestão Do Trabalho Em Saúde. Ijuí: Unijuí; 2007. p.143-171.

10. Booth A, Carroll C. Systematic searching for theory to inform systematic reviews: is it feasible? Is it desirable? Health Inf Libr J 2015; 32(3):220-235.

11. Bloom BS, Hastings JT, Madaus GF. Handbook on Formative and Summative Evaluation of Student Learning. Nova York: McGraw-Hill; 1971.

12. Medin DL, Ross BH, Markman AB. Cognitive Psycho$\log y .3^{a}$ ed. San Diego: Harcourt College Publishers; 2000.

13. Brasil. Ministério da Saúde (MS). Secretaria-Executiva. Núcleo Técnico da Política Nacional de Humanização. HumanizaSUS: Política Nacional de Humanização: A Humanização Como Eixo Norteador Das Práticas de Atenção e Gestão Em Todas as Instâncias Do SUS. Brasília: MS; 2004.

14. Brasil. Ministério da Saúde (MS). HumanizaSUS: Documento base para gestores e trabalhadores do SUS. $4^{\text {a }}$ ed. Brasília: MS; 2010

15. Moher D, Liberati A, Tetzlaff J, Altman DG, for the PRISMA Group. Preferred reporting items for systematic reviews and meta-analyses: the PRISMA statement. BMJ 2009; 339(21):b2535.

16. Moher D, Liberati A, Tetzlaff J, Altman DG. Principais itens para relatar Revisões sistemáticas e Meta-análises: A recomendação PRISMA. Epidemiol Serv Saude 2015; 24(2):335-342.

17. Rathert C, Mittler JN, Banerjee S, McDaniel J. Patientcentered communication in the era of electronic health records: What does the evidence say? Patient Educ Couns 2017; 100(1):50-64.
18. O'Malley AS, Draper K, Gourevitch R, Cross DA, Scholle SH. Electronic health records and support for primary care teamwork. J Am Med Inform Assoc 2015; 22(2):426-434.

19. Martins APOQ, Peres AM, Gil NLM, Ros CD, Lowen IMV, Gonçalves LS. Usabilidade do prontuário eletrônico em Unidades Básicas de Saúde. Cien Cuid Saude 2017; 16(2):1-7.

20. Schenk E, Schleyer R, Jones CR, Fincham S, Daratha KB, Monsen KA. Impact of Adoption of a Comprehensive Electronic Health Record on Nursing Work and Caring Efficacy: CIN Comput Inform Nurs 2018; 36(7):331-339.

21. Gomes PDAR, Farah BF, Rocha RS, Friedrich DB de C, Dutra HS. Electronic Citizen Record: An Instrument for Nursing Care / Prontuário Eletrônico do Cidadão: Instrumento Para o Cuidado de Enfermagem. Rev Pesqui Cuid Fundam Online 2019; 11(5):1226.

22. Alanazi B, Butler-Henderson K, Alanazi MR. The Role of Electronic Health Records in Improving Communication Between Health Professionals in Primary Healthcare Centres in Riyadh: Perception of Health Professionals. Stud Health Technol Inform 2019; 264:499-503.

23. Sockolow PS, Weiner JP, Bowles KH, Abbott P, Lehmann HP. Advice for Decision Makers Based on an Electronic Health Record Evaluation at a Program for All-inclusive Care for Elders Site. Appl Clin Inform 2011; 02(01):18-38.

24. Brasil. Ministério da Saúde (MS). Humaniza SUS: Documento Base Para Gestores e Trabalhadores do SUS [Internet]. $4^{\text {a }}$ ed. 2008 [acessado $2020 \mathrm{fev}$ 14]. Disponível em: http://bvsms.saude.gov.br/bvs/publicacoes/ humanizasus_gestores_trabalhadores_sus_4ed.pdf.

25. Godoy JSM, Gonçalves LS, Peres AM, Wolff LDG. O uso do prontuário eletrônico por enfermeiros em Unidades Básicas de Saúde brasileiras. J Health Inf 2012; 4(1):3-9.

26. Holmes ES. Atenção Básica como instrumento de apoio à tomada de decisão: avaliação de profissionais de saúde [dissertação]. João Pessoa: Universidade Federal da Paraíba; 2016.

27. Morrison Z, Fernando B, Kalra D, Cresswell K, Shei$\mathrm{kh}$ A. National evaluation of the benefits and risks of greater structuring and coding of the electronic health record: exploratory qualitative investigation. $J \mathrm{Am}$ Med Inform Assoc 2014; 21(3):492-500.

28. Soranz D, Pinto LF, Camacho LAB. Análise dos atributos dos cuidados primários em saúde utilizando os prontuários eletrônicos na cidade do Rio de Janeiro. Cirn Saude Colet 2017; 22(3):819-830.

29. Starfield B. Atenção primária: equilíbrio entre necessidades de saúde, serviços e tecnologia. UNESCO, MS; 2002.

Artigo apresentado em 26/10/2020

Aprovado em 18/12/2020

Versão final apresentada em 20/12/2020

Editores-chefes: Maria Cecília de Souza Minayo, Romeu Gomes, Antônio Augusto Moura da Silva 